\section{Microscopy with undetected photons in the mid-infrared}

\author{
Inna Kviatkovsky ${ }^{1 *}$, Helen M. Chrzanowski ${ }^{1}$, Ellen G. Avery ${ }^{2,3,4,5,6,7}$, \\ Hendrik Bartolomaeus ${ }^{2,3,4,5,6}$, Sven Ramelow ${ }^{1,8}$
}

\begin{abstract}
Owing to its capacity for unique (bio)-chemical specificity, microscopy with mid-infrared (IR) illumination holds tremendous promise for a wide range of biomedical and industrial applications. The primary limitation, however, remains detection, with current mid-IR detection technology often marrying inferior technical capabilities with prohibitive costs. Here, we experimentally show how nonlinear interferometry with entangled light can provide a powerful tool for mid-IR microscopy while only requiring near-IR detection with a silicon-based camera. In this proof-of-principle implementation, we demonstrate widefield imaging over a broad wavelength range covering 3.4 to $4.3 \mu \mathrm{m}$ and demonstrate a spatial resolution of $35 \mu \mathrm{m}$ for images containing 650 resolved elements. Moreover, we demonstrate that our technique is suitable for acquiring microscopic images of biological tissue samples in the mid-IR. These results form a fresh perspective for potential relevance of quantum imaging techniques in the life sciences.
\end{abstract}

\section{INTRODUCTION}

Mid-infrared (IR) imaging and microscopy are extensively used in various fields such as biology and medicine (1-6), environmental sciences (7), and microfluidics (8). Sensing with mid-IR light exploits the distinct rotational and vibrational modes of specific molecules (9). This spectral fingerprint can be used as a contrast mechanism for mid-IR imaging, circumventing the need for labeling. Such noninvasive and label-free imaging techniques are especially important for bioimaging procedures, as they permit the observation of largely unaltered living tissues. The current state-of-the-art mid-IR imaging technique is Fourier transform IR spectroscopic imaging (10). It heavily relies on IR technologies, namely, broadband IR sources and detectors. While the gap in technology and price between IR and visible sources is slowly closing (11), IR detection technology lags substantially behind its visible counterparts (12-14), such as complementary metal-oxide semiconductor (CMOS) and chargecoupled device technologies. Furthermore, IR detectors are costly and technically challenging, often requiring cryogenic cooling, and, moreover, are subject to severe export restrictions due to dual-use issues.

To bypass the need for IR detectors, techniques such as coherent Raman anti-Stokes scattering microscopy $(15,16)$ were developed. Here, the weakness of the Raman effect and intrinsic noise mechanism require high laser intensities and only permit slow point-bypoint scanning. Photothermal lens microscopy (17) and photoacoustic microscopy (18) in the mid-IR are two imaging modalities that are capable of imaging fresh biosamples with high spatial resolution. Nevertheless, raster scanning is required, and sample illumination

\footnotetext{
'Institut für Physik, Humboldt-Universität zu Berlin, Berlin, Germany. ${ }^{2}$ Experimental and Clinical Research Center, a cooperation of Charité-Universitätsmedizin Berlin and Max Delbrück Center for Molecular Medicine, Berlin, Germany. ${ }^{3}$ Charité-Universitätsmedizin Berlin, corporate member of Freie Universität Berlin, Humboldt-Universität zu Berlin, and Berlin Institute of Health, Berlin, Germany. ${ }^{4}$ Max Delbrück Center for Molecular Medicine in the Helmholtz Association, Berlin, Germany. ${ }^{5}$ DZHK (German Centre for Cardiovascular Research), partner site Berlin, Berlin, Germany. ${ }^{6}$ Berlin Institute of Health (BIH), Berlin, Germany. ${ }^{7}$ Freie Universität Berlin, Berlin, Germany. ${ }^{8}$ IRIS Adlershof, Humboldt-Universität zu Berlin, Berlin, Germany.

*Corresponding author. Email: innakv.ber@gmail.com
}

is comparatively invasive. Other approaches use frequency conversion to shift the detection frequency to the visible while still sensing in the IR region for the highly desired information. Up-conversion methods have demonstrated imaging in the near- and mid-IR (19-21), but conversion efficiency and the number of converted spatial modes remain a significant challenge, especially as they scale unfavorably with each other.

A markedly different approach uses the interference of an entangled photon pair with widely different wavelengths and requires neither laser sources nor detectors at the imaging wavelength. Photons at the idler wavelength are used for illumination but remain undetected. The initial proof of concept for wide-field imaging was demonstrated (22) at $1550 \mathrm{~nm}$ with moderate spatial resolution. There, the approach was based on induced coherence without induced emission $(23,24)$, but similar effects can be realized in general by nonlinear interferometers (25). It has also seen use, albeit in a single spatial mode regime for other modalities of sensing, such as spectroscopy (26), refractometry $(27)$, and optical coherence tomography $(28,29)$. In addition, during the preparation of this manuscript, we became aware of related work by Paterova et al. (30).

In this work, we show how highly multimode quantum nonlinear interferometry forms a powerful tool for microscopic imaging in the mid-IR using only a medium powered visible laser and a standard CMOS camera. We also derive explicit formulas for the field of view (FoV) and resolution for wide-field imaging with highly nondegenerate photon pairs, which are verified experimentally and numerically reproduced using a full quantum formalism (see Supplementary Materials). In our implementation, a nonlinear interferometer is formed by double passing a periodically poled potassium titanyl phosphate (ppKTP) crystal in a folded Michelson geometry (25). As the pump passes the crystal twice, it can generate a single pair of signal and idler photons via spontaneous parametric down-conversion (SPDC) in the first and/or second crystal. The signal and idler modes after the first pass of the crystal are aligned, such that when propagating back for the second pass, they perfectly overlap with the signal and idler modes for the second possibility for the biphoton generation. This results in indistinguishability and, thus, interference of single biphotons generated in the "first" and/or "second" crystal. The 
interference (of only one biphoton with itself) can be fully measured by solely looking at the signal photons with a CMOS camera. It reveals the phase and absorption an idler photon would experience after the first pass. No complex or cost-intensive components are required to realize such a setup.

In this work, the nonlinear crystal was engineered for highly nondegenerate signal and idler wavelengths. Using broadband phase matching (31), the idler wavelength can be selected in a large range between 3.4 and $4.3 \mu \mathrm{m}$ at room temperature, while the corresponding signal wavelength is in the 780 - to $820-\mathrm{nm}$ range. The strong spatial correlations between the signal and idler modes ensure that any distinguishing information obtained by the idler field between the first and second pass of the crystal will be encoded onto the interference of the near-IR light after the second crystal. This allows the simultaneous retrieval of both spatially resolved phase and amplitude information of a sample put into the idler arm. We characterize the mid-IR imaging properties of this system with an offthe-shelf CMOS camera. Moreover, the ability to manipulate the FoV and, accordingly, the system resolution is demonstrated. Specifically, using a $\times 10$ magnification, details down to $35 \mu \mathrm{m}$ are shown to be detected, which we use for acquiring microscopic images of a biological sample.

\section{RESULTS}

The experimental setup is detailed in Fig. 1. The initial characterization of the imaging technique was made in an unmagnified configuration, with both the end mirrors of the interferometer placed at the far-field of the crystal. The sample to be imaged is placed on the idler mirror. While the unmagnified configuration has limited spatial resolution, it provides a straightforward means to characterize the imaging capacity of the system. The lateral resolution was ascertained by measuring the spatial response to an edge knife (Fig. 2B), yielding $322 \pm 5 \mu \mathrm{m}$. The estimated number of spatial modes is $800 \pm 20$. In addition, a U.S. Air Force (USAF) clear path resolution target was illuminated (Fig. 2C). These values are consistent with the theoretical model (see Supplementary Materials) developed using a theoretical framework generalized from that of ghost imaging (32).

To demonstrate microscopy, a 10-fold magnification was realized via a telescope in the idler arm. Characterization of the magnified setup was performed in a similar manner, with the results summarized in Fig. 2. The obtained number of spatial modes is $655 \pm 57$. The system resolution of $35 \pm 5 \mu \mathrm{m}$ is below the smallest available line pair for our clear path resolution target (Fig. 2C).

The number of measured spatial modes in the unmagnified (magnified) realization is about $88 \%$ (72\%) of the theoretical value. We attribute this reduction in both optical arrangements to alignment imperfections, namely, in matching the corresponding imaging planes precisely, as well as chromatic aberrations. In addition, the smaller depth of focus in the magnified regime brings higher sensitivity to mismatch of imaging and sample planes, explaining the variation between the obtained number of modes in the unmagnified and magnified arrangements.

Our built-for-purpose SPDC source yields approximately $10^{8}$ pairs $/ \mathrm{s}$ within the filter bandwidth per $400 \mathrm{~mW}$ of pump light, which are distributed over around 18.000 pixels covering the FoV on the CMOS camera. This results in approximately 5000 photons per pixel per second, far above the intrinsic camera noise, and leads to the shot noise- and visibility-limited signal-to-noise ratios (SNRs) of the unmagnified (magnified) realization of around 25 (8) that we observe in our measurements. This corresponds to a resolvable transmission difference of $4 \%$ (12\%). The difference between the two realizations stems from the reduced visibility of the magnified arrangement,
A

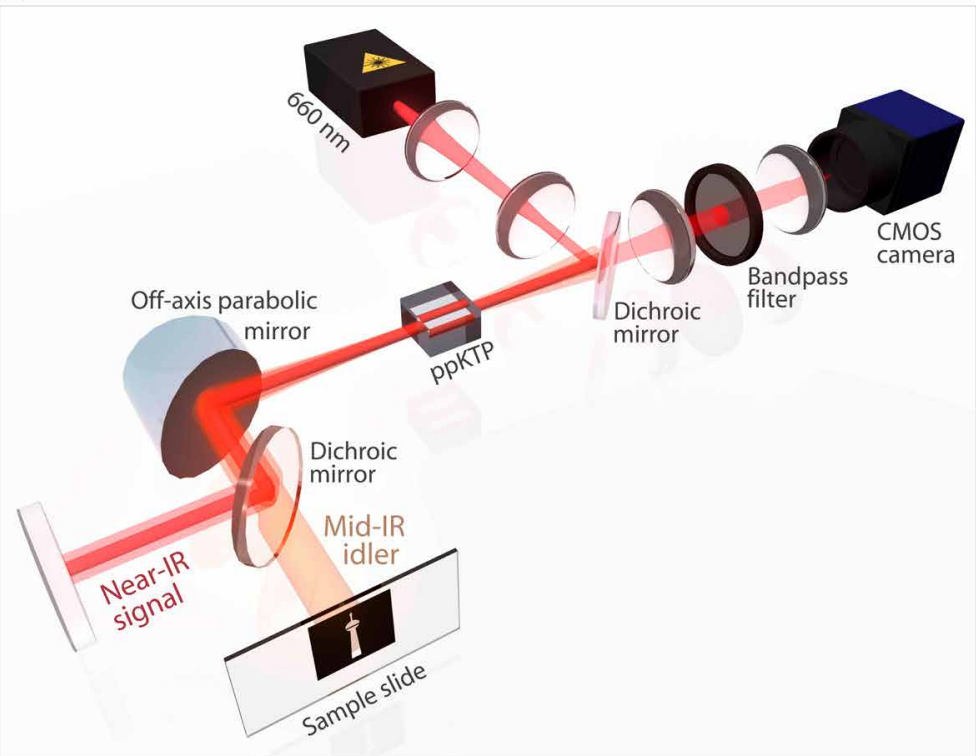

B

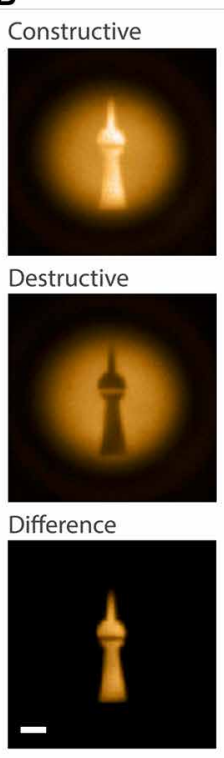

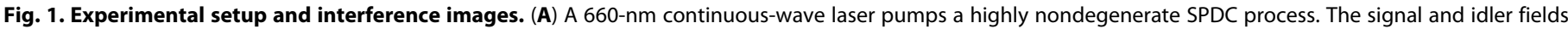

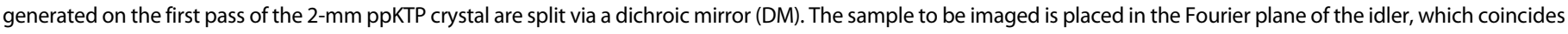

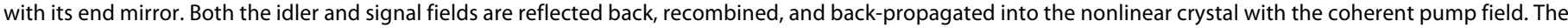

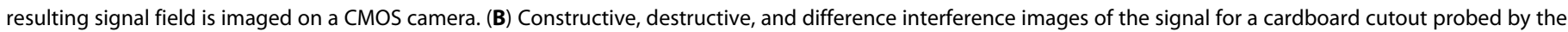
mid-IR idler. Scale bar, 2 
A
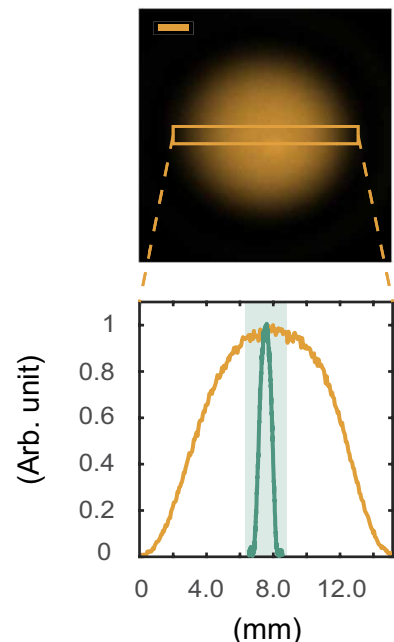

B
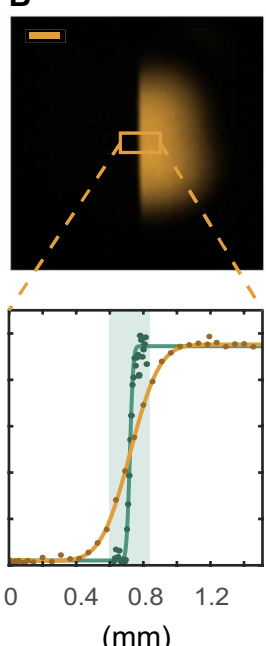

C
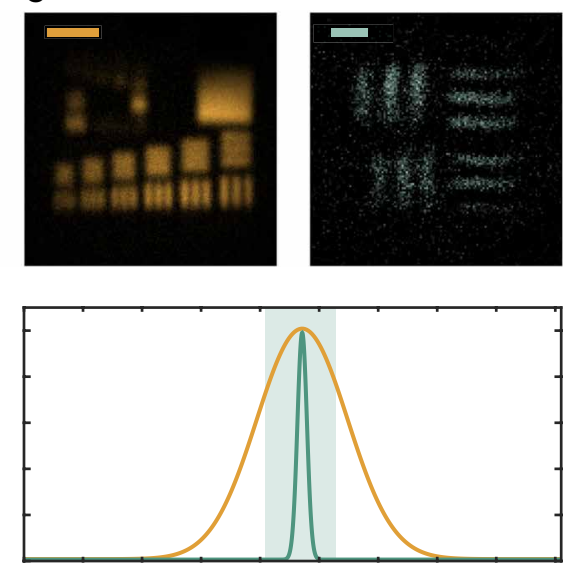

0

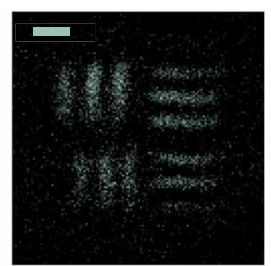

0.8
1.2

1.6

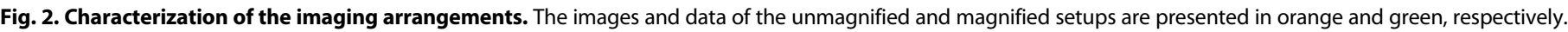

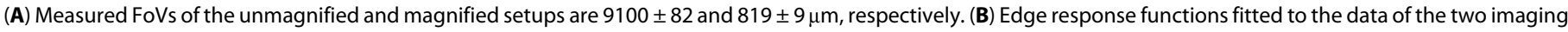

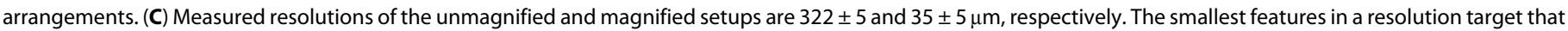

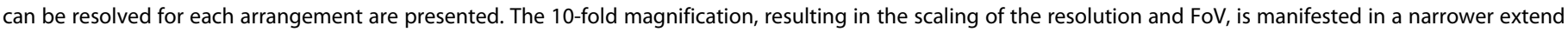

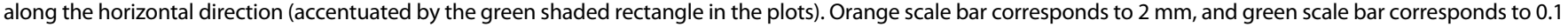

which we attribute to technical imperfections such as additional losses, alignment imperfections, and aberrations.

The highly broadband nature of the down-conversion source combined with the tight energy correlations shared between the signal and idler on the order of $1 \mathrm{MHz}$ (given by the pump laser linewidth) easily facilitates hyperspectral imaging. In our proof-of-concept demonstration, we use a tunable interference filter with $3.5-\mathrm{nm}$ bandwidth immediately before detection. Figure 3 presents transmission images obtained with the sample illumination spanning from 3.4 to $4.3 \mu \mathrm{m}$ with corresponding spectral selection of around $85 \mathrm{~nm}$ in the mid-IR. Note that the spectral resolution can be straightforwardly enhanced by narrower filtering and trading off signal strength.

Last, the potential of the presented method for realistic, biological samples was demonstrated using an unstained histology sample of a mouse heart (Fig. 4). The mid-IR images (Fig. 4B) were obtained by axially scanning the interferometer displacement inside the coherence length and extracting the visibility and phase of the interference signal for each pixel. This eliminates any ambiguity between loss and destructive interference (owing to a nonuniform phase front) that may arise in a single-shot measurement. It also permits straightforward reconstruction of the wide-field phase-contrast images (Fig. 4C). In the resulting images of the mouse heart in Fig. $4 \mathrm{C}$, ii the arrows indicate a portion of the endocardium, the innermost layer that lines the heart ventricles. This can be seen as a dark purple region indicating high absorption. This layer separates the ventricle itself and the myocardium, the cardiac muscle that constitutes the bulk portion of the heart tissue. The images are a testament to the high tolerance of the presented imaging method to loss and scattering.

\section{DISCUSSION}

We have demonstrated the capacity of mid-IR imaging via nonlinear interferometry for real-world imaging tasks, requiring only cost- efficient components. Via careful addition of a magnification step, we demonstrate imaging of feature sizes down to $35 \mu \mathrm{m}$.

Furthermore, owing to the use of a broadband SPDC, the extension to hyperspectral imaging is uncomplicated. To demonstrate the real-world promise of this method for nondestructive biological sensing, we have imaged a wet biological sample with a low sample illumination of less than $20 \mathrm{pW}$. Given this, one must contemplate what underlies the (perhaps unexpected) efficacy of the presented technique. First, because of the SPDC process, for every detected signal photon exists a corresponding idler photon, and owing to the intrinsic unity efficiency of the nonlinear interferometer, any image information carried by that idler photon can be transferred perfectly to the signal photon. Thus, in the absence of loss and mode mismatch, one can effectively consider the "mid-IR information detection efficiency" to be determined by the efficiency and noise properties of the near-IR camera used to image the signal field, with modern silicon technology permitting near shot noise-limited images with only a few 1000 of photons per pixel per second. Moreover, this technique has an interesting intrinsic scaling advantage when compared with up-conversion imaging approaches. Consider increasing the number of available modes by a factor of $N^{2}$ : Both techniques would require factor $N$ increase in the pump laser waist (and thus the crystal to accommodate it). However, to maintain a constant SNR, nonlinear interferometry requires an $N^{2}$ increase in the pump power, whereas up-conversion imaging would demand an increase of $N^{3}$.

While the spatial resolution presented here with our basic optical layout is larger than the resolutions anticipated by the state-of-theart mid-IR systems ( 1 to $10 \mu \mathrm{m}$ ), the extension to increased imaging capacity is straightforward. The number of available spatial modes is currently limited by the size of the pump waist and, thus, the crystal aperture. With an increase in the crystal aperture from 1 to $4 \mathrm{~mm}$, the number of available spatial modes will grow from $\approx 750$ to 12,000 . Naturally, this 16 -fold increase comes at the expense of the 

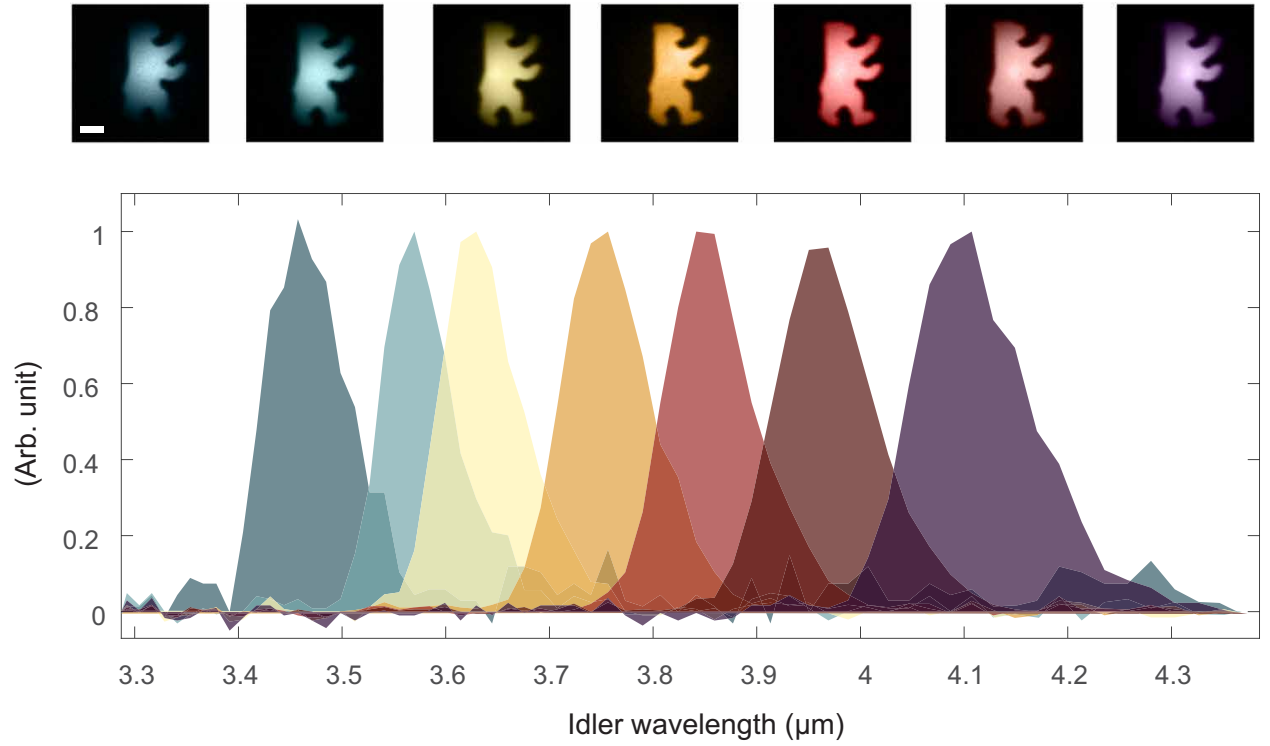

Fig. 3. Multispectral imaging. Obtained signal transmission images for varying mid-IR illumination wavelengths. Scale bar, $2 \mathrm{~mm}$. The spectra were recorded at the signal wavelength with a grating spectrometer and converted to the corresponding mid-IR wavelength.

A

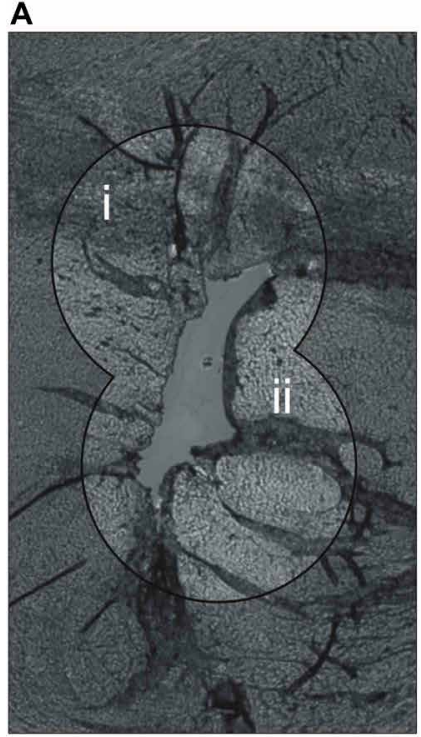

B
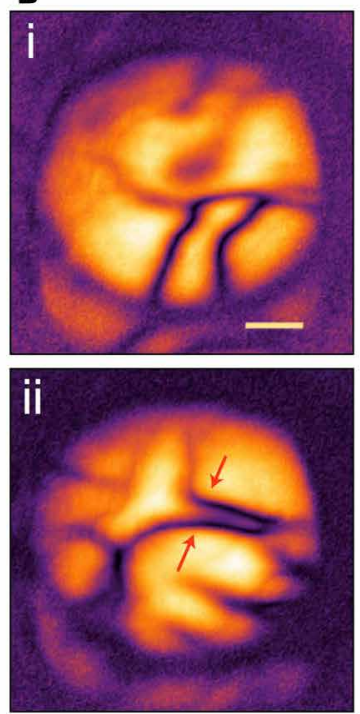

C
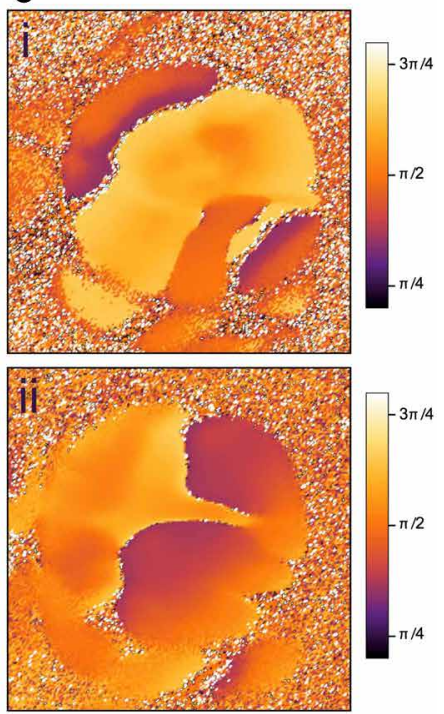

Fig. 4. Bioimaging. Histology sample of a mouse heart with (A) bright-field microscopy with visible light for illustration of the part of the sample we investigated with our method. (B and C) Mid-IR microscopy of the same sample with undetected photons for absorption (B) and phase (C) imaging. Scale bar, 200

per pixel illumination. Small improvements, however, such as optimized camera illumination (2.5 pixels per resolved mode from the existing 5) and increased exposure time (from 1 to $4 \mathrm{~s}$ ) will return us to the current per pixel illumination, while further gains remain accessible via crystal optimization and increased pump power. These improvements, coupled with more sophisticated approaches to magnification, will yield imaging capacities at the state of the art while surpassing its speed by leveraging silicon camera technology. In addition, the latter also ensures that the pixel number of the camera itself is no longer a potential bottleneck, since sensitive megapixel CMOS cameras are widely available.

Crucially for applications in hyperspectral imaging, the use of a purpose-engineered broadband SPDC source (31) allows a broad wavelength range to probe the sample simultaneously. Harnessing the potential for high spectral selectivity-fundamentally limited only by the bandwidth of the pump laser-will fully unlock the potential of this technique. For example, with efficient extraction of the spectral information and a larger crystal aperture, a mid-IR hyperspectral 
microscopy image with a $10-\mu \mathrm{m}$ spatial resolution and 250 spectral modes could be achieved within a few minutes (see the Supplementary Materials).

In conclusion, we have experimentally demonstrated that nonlinear interferometry with entangled photons provides a powerful and cost-effective technique for microscopy in the mid-IR, harnessing the maturity of silicon-based near-IR detection technology to allow mid-IR imaging with exceptionally low-light-level illumination. We have shown how this technique can be readily extended to hyperspectral imaging across nearly $1 \mu \mathrm{m}$. A practical biological sample was imaged with quantum light, revealing morphological features with high resolution. Our results pave the way for broadband, hyperspectral mid-IR microscopy with fast, wide-field imaging capabilities, enabling far-ranging applications in biomedical imaging.

\section{MATERIALS AND METHODS Experimental setup}

A 660-nm continuous-wave solid-state laser delivers the pump beam for the experiment. Using a telescopic arrangement and a dichroic mirror (DM), the pump illuminates the ppKTP crystal with a $430-\mu \mathrm{m}$ waist, maximally covering the $1 \times 2-\mathrm{mm}$ crystal aperture. The ppKTP crystal is quasi-phase matched for a collinear type 0 process, specifically engineered to produce highly nondegenerate photon pairs. The photon pairs are ultrabroadband due to group velocity matching, with an idler spectrum from 3.38 to $4.29 \mu \mathrm{m}$. The emerging signal and idler (and pump) fields are subsequently collimated (focused) by an off-axis parabolic mirror (OPM). Using a DM, the idler is then split from the signal and pump and is incident on a sample placed on the end mirror of the interferometer. The end mirrors of the interferometer (and thus the object) are placed in the Fourier plane of the crystal. The use of lenses between the OPM and the idler and signal end mirrors is primarily for obtaining the desired FoV on the sample and for matching the idler and signal interferometric arms, respectively. In our magnified setup, a 10-fold magnification is introduced in the idler arm via a telescopic arrangement. The pump is reflected using a cold mirror at the focal length of the OPM. The reflected idler, signal, and pump fields back-propagate through the same path, back into the crystal, with the pump again generating signal and idler fields. Before detection, the signal field is filtered using a 3.5-nm bandpass filter. The Fourier plane of the resulting signal field is then imaged on a CMOS camera. The interferometer arm lengths are required to be matched to within the coherence length of the detected light. For the spectrally filtered image, this length is typically $100 \mu \mathrm{m}$. The Michelson-type interferometer geometry strongly simplifies the alignment process.

\section{Slide preparation}

Nine- to 12-week-old C57BL/6J mice were euthanized by cervical dislocation. For formalin fixation, hearts were removed, rinsed in ice-cold saline, and placed in $4 \%$ formalin. After 48 to 72 hours of fixation, the tissue was rinsed with phosphate-buffered saline and, lastly, embedded in paraffin. The paraffin-embedded hearts were cut in transverse sections to a thickness of 2 to $3 \mu \mathrm{m}$ and transferred to a low-e slide. Slides were stored at room temperature.

\section{SUPPLEMENTARY MATERIALS}

Supplementary material for this article is available at http://advances.sciencemag.org/cgi/ content/full/6/42/eabd0264/DC1

\section{REFERENCES AND NOTES}

1. J. T. Kwak, S. M. Hewitt, S. Sinha, R. Bhargava, Multimodal microscopy for automated histologic analysis of prostate cancer. BMC Cancer 11, 62 (2011).

2. C. L. Evans, X. S. Xie, Coherent anti-stokes raman scattering microscopy: Chemical imaging for biology and medicine. Annu. Rev. Anal. Chem. 1, 883-909 (2008).

3. C. L. Evans, X. Xu, S. Kesari, X. S. Xie, S. T. C. Wong, G. S. Young, Chemically-selective imaging of brain structures with CARS microscopy. Opt. Express 15, 12076-12087 (2007).

4. G. Bellisola, C. Sorio, Infrared spectroscopy and microscopy in cancer research and diagnosis. Am. J. Cancer Res. 2, 1-21 (2012).

5. K. Potter, L. H. Kidder, I. W. Levin, E. N. Lewis, R. G. S. Spencer, Imaging of collagen and proteoglycan in cartilage sections using Fourier transform infrared spectral imaging. Arthritis Rheum. 44, 846-855 (2001).

6. L. M. Miller, M. W. Bourassa, R. J. Smith, Ftir spectroscopic imaging of protein aggregation in living cells. Biochim. Biophys. Acta Biomembr. 1828, 2339-2346 (2013).

7. A. S. Tagg, M. Sapp, J. P. Harrison, J. J. Ojeda, Identification and quantification of microplastics in wastewater using focal plane array-based reflectance micro-FT-IR imaging. Anal. Chem. 87, 6032-6040 (2015).

8. A. F. Chrimes, K. Khoshmanesh, P. R. Stoddart, A. Mitchell, K. Kalantar-zadeh, Microfluidics and Raman microscopy: Current applications and future challenges. Chem. Soc. Rev. 42, 5880-5906 (2013).

9. R. A. Shaw, H. H. Mantsch, Vibrational biospectroscopy: From plants to animals to humans. A historical perspective. J. Mol. Struct. 480, 1-13 (1999).

10. R. Bhargava, Infrared spectroscopic imaging: The next generation. Appl. Spectrosc. $\mathbf{6 6}$ 1091-1120 (2012).

11. A. Godard, Infrared (2-12 $\mu \mathrm{m})$ solid-state laser sources: A review. Comptes Rendus Physique 8, 1100-1128 (2007).

12. A. Rogalski, Infrared detectors: An overview. Infrared Phys. Technol. 43, 187-210 (2002).

13. D. A. Scribner, M. R. Kruer, J. M. Killiany, Infrared focal plane array technology. Proc. IEEE 79, 66-85 (1991).

14. A. Rogalski, P. Martyniuk, M. Kopytko, Challenges of small-pixel infrared detectors: A review. Rep. Prog. Phys. 79, 046501 (2016).

15. M. Müller, J. M. Schins, Imaging the thermodynamic state of lipid membranes with multiplex cars microscopy. J. Phys. Chem. B 106, 3715-3723 (2002).

16. J.-X. Cheng, X. S. Xie, Vibrational spectroscopic imaging of living systems: An emerging platform for biology and medicine. Science 350, aaa8870 (2015).

17. D. Zhang, C. Li, C. Zhang, M. N. Slipchenko, G. Eakins, J.-X. Cheng, Depth-resolved mid-infrared photothermal imaging of living cells and organisms with submicrometer spatial resolution. Sci. Adv. 2, e1600521 (2016).

18. J. Shi, T. T. W. Wong, Y. He, L. Li, R. Zhang, C. S. Yung, J. Hwang, K. Maslov, L. V. Wang, High-resolution, high-contrast mid-infrared imaging of fresh biological samples with ultraviolet-localized photoacoustic microscopy. Nat. Photon. 13, 609-615 (2019).

19. R. Demur, R. Garioud, A. Grisard, E. Lallier, L. Leviandier, L. Morvan, N. Treps, C. Fabre, Near-infrared to visible upconversion imaging using a broadband pump laser. Opt. Express 26, 13252-13263 (2018).

20. J. S. Dam, P. Tidemand-Lichtenberg, C. Pedersen, Room-temperature mid-infrared single-photon spectral imaging. Nat. Photon. 6, 788-793 (2012).

21. S. Junaid, S. C. Kumar, M. Mathez, M. Hermes, N. Stone, N. Shepherd, M. Ebrahim-Zadeh, P. Tidemand-Lichtenberg, C. Pedersen, Video-rate, mid-infrared hyperspectral upconversion imaging. Optica 6, 702-708 (2019).

22. G. B. Lemos, V. Borish, G. D. Cole, S. Ramelow, R. Lapkiewicz, A. Zeilinger, Quantum imaging with undetected photons. Nature 512, 409-412 (2014).

23. L. J. Wang, X. Y. Zou, L. Mandel, Induced coherence without induced emission. Phys. Rev. A 44, 4614-4622 (1991)

24. X. Y. Zou, L. J. Wang, L. Mandel, Induced coherence and indistinguishability in optical interference. Phys. Rev. Lett. 67, 318-321 (1991).

25. M. V. Chekhova, Z. Y. Ou, Nonlinear interferometers in quantum optics. Adv. Opt. Photon. 8, 104-155 (2016).

26. D. A. Kalashnikov, A. V. Paterova, S. P. Kulik, L. A. Krivitsky, Infrared spectroscopy with visible light. Nat. Photon. 10, 98-101 (2016).

27. A. Paterova, H. Yang, C. An, D. Kalashnikov, L. Krivitsky, Measurement of infrared optical constants with visible photons. New J. Phys. 20, 043015 (2018).

28. A. Vallés, G. Jiménez, L. J. Salazar-Serrano, J. P. Torres, Optical sectioning in induced coherence tomography with frequency-entangled photons. Phys. Rev. A 97, 023824 (2018).

29. A. V. Paterova, H. Yang, C. An, D. A. Kalashnikov, L. A. Krivitsky, Tunable optical coherence tomography in the infrared range using visible photons. Quantum Sci. Technol. 3, 025008 (2018).

30. A. V. Paterova, S. M. Maniam, H. Yang, G. Grenci, L. A. Krivitsky, Hyperspectral infrared microscopy with visible light. arXiv:2002.05956 [physics.optics] (14 February 2020).

31. A. Vanselow, P. Kaufmann, H. M. Chrzanowski, S. Ramelow, Ultra-broadband SPDC for 
32. P.-A. Moreau, E. Toninelli, P. A. Morris, R. S. Aspden, T. Gregory, G. Spalding, R. W. Boyd, M. J. Padgett, Resolution limits of quantum ghost imaging. Opt. Express 26, 7528-7536 (2018).

33. E. Brambilla, A. Gatti, M. Bache, L. A. Lugiato, Simultaneous near-field and far-field spatial quantum correlations in the high-gain regime of parametric down-conversion. Phys. Rev. A 69, 023802 (2004).

34. M. Katz, D. Eger, M. B. Oron, A. Hardy, Refractive dispersion curve measurement of $\mathrm{KTiOPO}_{4}$ using periodically segmented waveguides and periodically poled crystals. J. Appl. Phys. 90, 53-58 (2001).

35. T. Y. Fan, C. E. Huang, B. Q. Hu, R. C. Eckardt, Y. X. Fan, R. L. Byer, R. S. Feigelson, Second harmonic generation and accurate index of refraction measurements in flux-grown $\mathrm{KTiOPO}_{4}$. Appl. Opt. 26, 2390-2394 (1987).

Acknowledgments: We would like to thank A. Hochrainer, M. Lahiri, A. Zeilinger, G. B. Lemos, and R. Lapkiewicz for the fruitful discussions about the theory and characterization of spatial resolution in imaging with undetected photons. Funding: The work was funded by Deutsche
Forschungsgemeinschaft (RA 2842/1-1). Author contributions: S.R. conceived the idea and supervised the project. I.K. and S.R. designed the experiment. I.K. carried out the experiment with assistance from H.M.C. and S.R. I.K. and H.M.C. analyzed the data. I.K., H.M.C., and S.R. developed the theoretical analysis. E.G.A. and H.B. prepared the biological samples and assisted in interpreting the results. All authors contributed to the preparation of the manuscript. Competing interests: The authors declare that they have no competing interests. Data and materials availability: All data needed to evaluate the conclusions in the paper are present in the paper and/or the Supplementary Materials. Additional data related to this paper may be requested from the authors.

Submitted 28 May 2020

Accepted 2 September 2020

Published 14 October 2020

$10.1126 /$ sciadv.abd0264

Citation: I. Kviatkovsky, H. M. Chrzanowski, E. G. Avery, H. Bartolomaeus, S. 


\section{ScienceAdvances}

\section{Microscopy with undetected photons in the mid-infrared}

Inna Kviatkovsky, Helen M. Chrzanowski, Ellen G. Avery, Hendrik Bartolomaeus and Sven Ramelow

Sci Adv 6 (42), eabd0264.

DOI: $10.1126 /$ sciadv.abd0264

ARTICLE TOOLS

SUPPLEMENTARY MATERIALS

REFERENCES

PERMISSIONS http://advances.sciencemag.org/content/6/42/eabd0264

http://advances.sciencemag.org/content/suppl/2020/10/09/6.42.eabd0264.DC1

This article cites 34 articles, 2 of which you can access for free $\mathrm{http}: / /$ advances.sciencemag.org/content/6/42/eabd0264\#BIBL

http://www.sciencemag.org/help/reprints-and-permissions

Science Advances (ISSN 2375-2548) is published by the American Association for the Advancement of Science, 1200 New York Avenue NW, Washington, DC 20005. The title Science Advances is a registered trademark of AAAS.

Copyright ( 2020 The Authors, some rights reserved; exclusive licensee American Association for the Advancement of Science. No claim to original U.S. Government Works. Distributed under a Creative Commons Attribution NonCommercial License 4.0 (CC BY-NC). 